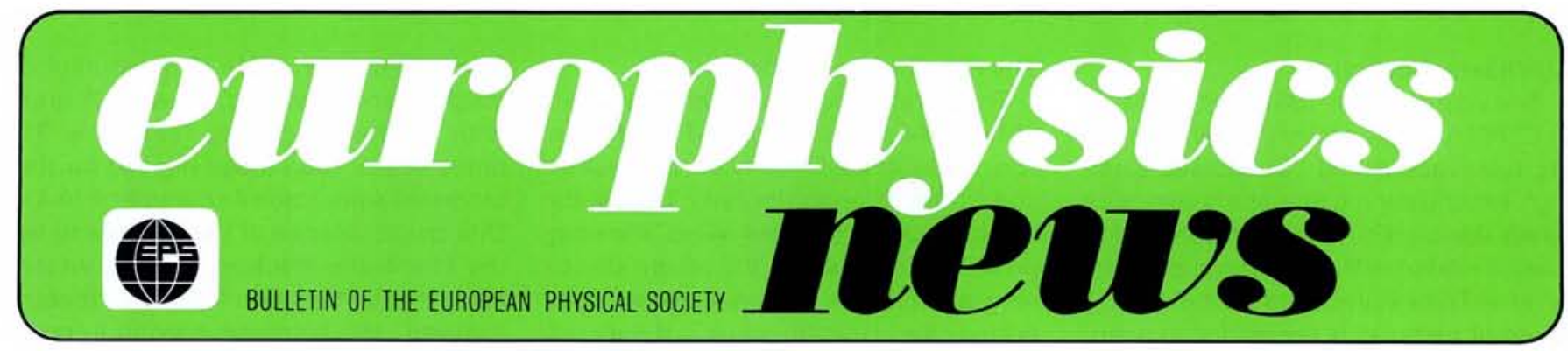

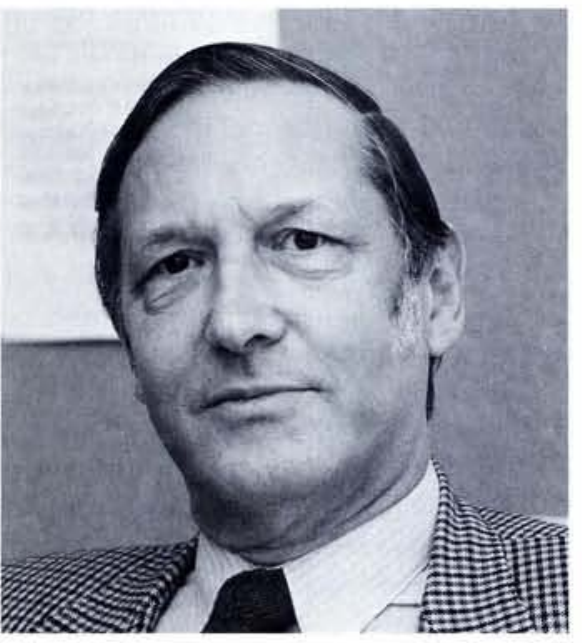

Within minutes of the arrival of the following telegram, TV monitor screens all over CERN were transmitting the news.

STOCKHOLM, OCTOBER 17, 1984

THE ROYAL SWEDISH ACADEMY OF SCIENCES HAS TODAY DECIDED TO AWARD THE NOBEL PRIZE IN PHYSICS FOR 1984 JOINTLY TO

PROFESSOR CARLO RUBBIA, CERN,

GENEVA, SWITZERLAND

AND

DR SIMON VAN DER MEER, CERN,

GENEVA, SWITZERLAND

FOR THEIR DECISIVE CONTRIBU-

TIONS TO THE LARGE PROJECT, WHICH LED TO THE DISCOVERY OF THE FIELD PARTICLES W AND Z, COMMUNICATORS OF WEAK INTERACTIONS

THE ROYAL SWEDISH ACADEMY OF SCIENCES

\section{INFORMATION DEPARTMENT}

Characteristically, when the message arrived, Rubbia, en route to a conference in Trieste was stranded at Milan Airport by a strike, and van der Meer was sitting quietly in his $12 \mathrm{~m}^{2}$ office.

The news of the award, although not entirely unexpected, spread even faster than that of the first $W$ candidates less than two years earlier. The broad smiles to be seen all over CERN expressed the genuine pleasure felt for the Laureates and the feeling of personal involvement.

S. van der Meer (left) and Carlo Rubbia

\section{4 Nobel Prize in Physics}

\section{Roy Billinge, CERN}

Photo CERN

Photo CERN

It is undeniable that the "large project" and the search for the intermediate vector bosons had involved the whole of CERN - firing the imagination to an extent that no other project has equalled. At the official celebration for the CERN staff on Friday, 19 October, Carlo and Simon both underlined the contribution of all the staff. Nevertheless, the Swedish Academy in its wisdom recognized that these two individuals made "decisive contributions" and while it may often be difficult in "Big Science" to draw a line and identify one or two key people, in this case the roles of the two Laureates were clearly in a class of their own.

As with all flairs of genius, their ideas - after the event - seem rather simple.

\section{Historical Background}

The milieu of High Energy Physics and Accelerators is one in which there is constantly a multitude of new ideas, theories, techniques being proposed sometimes developed, sometimes abandoned. The possibility of achieving very high centre of mass energies by colliding counter-rotating beams of particles has tempted physicists for many years, and led to the successful construction and operation of the Intersecting Storage Rings at CERN.

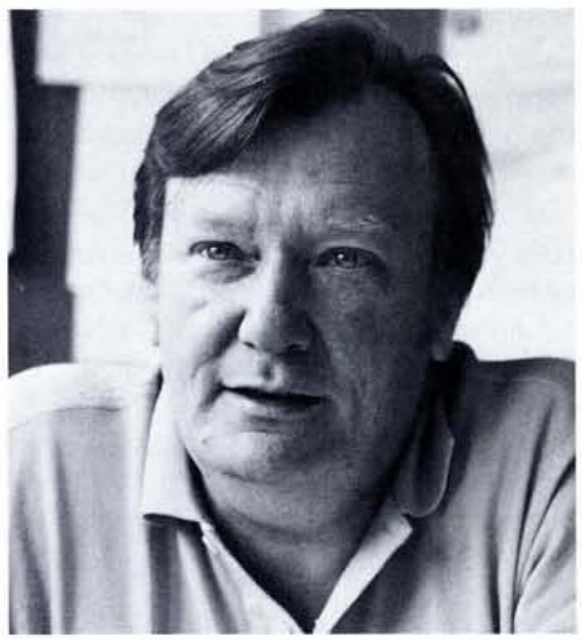

In 1966, Budker and Skrinsky at Novosibirsk suggested that high luminosity proton-antiproton collisions in a single magnet ring might become feasible by means of a technique called electron cooling, which could be used to obtain a high density antiproton beam. The technique consists of mixing a high current electron beam with the antiproton beam. By precise matching of the velocities of the two beams, the Coulomb interaction tends to equalize their "temperatures" so that an electron beam with very little transverse motion, will reduce that of the antiprotons whose density is thus increased.

\section{Contents}

1984 Nobel Prize in Physics 1

Macroscopic Quantum Effects in Disordered Systems

Small Particles in Gas Suspension 6

Extragalactic Distance Scale and the Hubble Constant

High Field Magnet Laboratory at Nijmegen

Society News 16 


\section{Stochastic Cooling}

Two years later, in 1968, van der Meer at CERN proposed a very different cooling technique called "Stochastic Cooling". Essentially the process depends on being able continuously to resolve information related to the position and velocity of a finite number of particles. If a beam of particles is circulating in a storage ring, a sensitive detector located at one point on the circumference can be made to pick up the statistical fluctuations of the "centre of gravity" of the beam and, by amplifying this signal and applying it to a corrector elsewhere in the ring with the appropriate phase, the detected fluctuation can be reduced.

In order to understand how the process works, we first consider the simple case in which the detector - amplifier - corrector chain can act sufficiently fast to treat each particle individually. In other terms, the bandwidth of the system $(W)$ equals the product of the number of particles $(N)$ and their frequency of revolution $\left(f_{r}\right)$

i.e.

$$
W=N f_{r}
$$

In this case, the errors could in principle be eliminated in a single revolution or, in a time given by

$$
\text { i.e. } \quad \begin{aligned}
\tau & =1 / f_{r} \\
\tau & =N / W
\end{aligned}
$$

This trivial example already indicates the correct proportionality between cooling time, number of particles and bandwidth.

In practice, the smallest time interval which can be resolved by the system contains many particles, so that correction is based on the average error of the sample thus reducing the fluctuation of its centre of gravity. This could only lead to a very small amount of "cooling". However, besides having a range of transverse oscillation amplitudes, the particles cover a range of energy and therefore revolution frequency. This leads to a given time-slice (sample) of the beam having a changing population of particles. The "mixing" in this way leads to samples which evolve, and the cooling process can be progressive.

Perhaps one of the better analogies invoked to describe the physical process, is one due to van der Meer himself. We imagine a box inside which weights, suspended from springs are oscillating vertically with different frequencies and amplitudes. By shaking the box up and down in just the right way, it is possible to bring all the weights to rest. Finding "just the right way" of shaking a stored beam of particles to steadily increase its density is the subject of Stochastic Cooling.

\section{The Hunt for the Bosons}

Theoretical and experimental studies of both electron cooling and stochastic cooling continued mainly at Novosibirsk and CERN, through the early 1970's. By 1976 , both techniques were showing promising results and the recent discovery at CERN of neutral currents supported the "electro-weak" theory of Glashow, Salam and Weinberg which promised the synthesis of two of the known forces. At this time also the discovery of charmed particles caused great excitement in particle physics and was soon followed by the approval of projects for the higher energy electronpositron colliders PEP and PETRA. What was needed to confirm the electro-weak theory was to find the $\mathrm{W}$ and $\mathrm{Z}$ bosons whose masses were predicted to be close to 80 and $90 \mathrm{GeV} / \mathrm{c}^{2}$ respectively.

However, producing such massive objects would require an electron-positron collider of up to $100 \mathrm{GeV}$ centre of mass energy or, due to the composite structure of the proton, a hadron collider with about five times this energy. Out of this sea of speculation, theories and ideas emerged Rubbia's bold and imaginative idea, to collect and cool sufficient antiprotons to permit the use of either the Main Ring at Fermilab or the recently commissioned SPS at CERN as protonantiproton collider with centre of mass energies approaching $600 \mathrm{GeV}$.

During 1976, working groups examined the technical aspects of such schemes and the physics potential. As a result, CERN decided to pursue two courses of action in parallel. One was to construct rapidly, a small experimental ring (ICE) to study both electron and stochastic cooling; the other was to set up a study group to prepare a design for a $\bar{p} \bar{p}$ facility using the SPS as a storage ring. Initially the study group produced a proposal using two separate rings for collecting and cooling antiprotons, based on electron cooling. However many experimental tests in the ISR, further theoretical developments and most importantly the proposal of a faster and more efficient method of momentum cooling (the Thorndhal method) indicated the possibility of a solution based entirely on stochastic cooling and stacking with a single DC operated accumulator ring. The potential savings in cost and complexity led us to adopt this scheme even though the cooling required represented an extrapolation of a factor of 1000 times over the ISR experiments.

The overall increase in antiproton density required in the proposal was a factor of just over $10^{9}$ relative to that from the production target.
In the course of 1978 encouraging results were obtained from the ICE ring, with stochastic cooling rates only 30 times slower than those needed for the proposed Antiproton Accumulator (AA). One crucial feature of the AA was to be the stochastic stacking process which could not be tested in ICE. The process involved: simultaneous cooling in both transverse directions and progressively increasing the longitudinal density by a factor of $10^{4}$ whilst moving the particles into the dense stack "core". It is typical of Simon, that he studied this whole process in every detail, constructed computational models and developed the theory until he became convinced that it would work. From there on no further discussion was needed.

Another serious reservation at that time was the feasibility of being able to find the $W$ and $Z$ particles amongst the vast amounts of data which would have to be recorded by the experiment. For example, the cross-section for producing a $Z_{0}$ decaying into an electron-positron pair was predicted to be $10^{-9}$ of the total reaction cross-section. Carlo, however, never seemed to have any doubts. Having acquired his formidable experience in experimental physics beginning at the SC then on the PS, the ISR and recently the SPS, his confidence was indeed well founded. Amongst all this, the most crucial was his experience on the ISR without which, he admits, the correct design of the UA1 experiment, would not have been possible.

In July 1978 the $p \bar{p}$ project was approved and by July 1980 the AA was completed. First $\mathrm{p} \overline{\mathrm{p}}$ collisions were obtained in the SPS in 1981 and in 1982 the first $W$ boson candidate events were found, to be followed in 1983 by $Z_{0}$ events and in 1984 - The Nobel Prize for Physics.

\section{The Laureates}

Both are men of outstanding originality and imagination. Rubbia's boundless energy and enthusiasm were an essential component to get his ideas adopted at CERN. Van der Meer's quiet and thorough study of stochastic cooling and stacking led to our hope - and later conviction that it could all be made to work. Bringing together a team of 150 physicists from all over Europe and designing an experiment to pick out the one $Z_{0}$ amongst one thousand million other events has stimulated a world wide interest in Hadron Colliders for the future.

\section{REFERENCES}

Brianti G. and Gabathuler E., Intermediate Vector Bosons,

Europhysics News, 14 (1983) 10. 